\title{
Role of Gas Viscosity for Shale Gas Percolation
}

\author{
Xiaoming Wang, ${ }^{1,2,3}$ Junbin Chen $\mathbb{D}^{2,3}$ Dazhong Ren, ${ }^{2,3}$ and Zhaolong Shi ${ }^{2,3}$ \\ ${ }^{1}$ College of Geology and Environment, Xi'an University of Science and Technology, Xi'an, Shaanxi 710054, China \\ ${ }^{2}$ Shaanxi Key Laboratory of Well Stability and Fluid \& Rock Mechanics in Oil and Gas Reservoirs, Xi'an Shiyou University, Xi'an, \\ Shaanxi 710065, China \\ ${ }^{3}$ College of Petroleum Engineering, Xi'an Shiyou University, Xi'an, Shaanxi 710065, China
}

Correspondence should be addressed to Junbin Chen; 1391927626@qq.com

Received 11 August 2020; Revised 12 September 2020; Accepted 16 September 2020; Published 30 September 2020

Academic Editor: Jinze Xu

Copyright (C) 2020 Xiaoming Wang et al. This is an open access article distributed under the Creative Commons Attribution License, which permits unrestricted use, distribution, and reproduction in any medium, provided the original work is properly cited.

\begin{abstract}
Viscosity is an important index to evaluate gas flowability. In this paper, a double-porosity model considering the effect of pressure on gas viscosity was established to study shale gas percolation through reservoir pressure, gas velocity, and bottom hole flowing pressure. The experimental results show that when pressure affects gas viscosity, shale gas viscosity decreases, which increases the percolation velocity and pressure drop velocity of the free state shale gas in matrix and fracture systems. And it is conducive to the desorption of adsorbed shale gas and effectively supplemented the bottom hole flowing pressure with the pressure wave propagation range and velocity increasing, so that the rate of pressure drop at the bottom of the well slows down, which makes the time that bottom hole flowing pressure reaches stability shortened. Therefore, the gas viscosity should be fully considered when studying the reservoir gas percolation.
\end{abstract}

\section{Introduction}

As one of the fast growing activities in the natu ral gas industry, shale gas development is playing an important role in the current energy mix of the world's growing energy demand $[1,2]$. Shale gas is a special kind of gas, which mainly exists in the multiscale reservoir space dominated by organic shale in three states: free state, adsorbed state, and dissolved state [3-5]. In the meanwhile, the percolation law of shale gas is very different from that of conventional gas reservoirs because of the various percolation patterns in the multiscale reservoir [6]. Therefore, the underlying flow mechanism through these multiporosity and multipermeability systems is complicated and poorly understood [7]. However, gas percolation is the core problem of gas exploitation, which is of great significance for the scientific and efficient development of unconventional tight shale reservoirs. In order to study the percolation phenomenon of shale gas, it is necessary to study from its essence, and viscosity is one of the important factors affecting percolation.
Shale gas is composed of molecules, and the macroscopic properties of its fluid are essentially determined by the motion of molecules. The characteristic of friction force between fluid molecules in actual fluid flow is called viscosity, and the physical quantity that measures the stickiness of fluid is viscosity, which happens to be one of the migration properties of fluid and affects the fluid percolation velocity [8]. Therefore, in order to interpret the percolation of shale gas fundamentally, it is necessary to consider the gas viscosity when evaluating the percolation of shale gas in the reservoir.

At present, the percolation law of shale gas is studied mainly from the following aspects. Firstly, the multiscale pore structure of the shale gas reservoir after fracturing is a key factor affecting shale gas percolation. It was found that different gas migration mechanisms exist in different migration channels. Considering the complex structures, such as nanopore, micronanopore, natural fracture, and artificial fracture, the Knudsen number is used to divide the flow among different reservoir scales, so that the shale gas percolation can be described from microscale to macroscale accurately $[9,10]$. Secondly, the selection of the percolation 
mathematical model is also a key point to describe and analyze the process of shale gas percolation. It is found that different mathematical models describe different communication situations between fractures and the reservoir matrix, such as the dual media model, continuum model, fractal induced fracture network distribution (FIFND) model, and three-dimensional numerical model [11-14], which greatly affect the shale gas percolation effect and the accuracy of shale gas percolation description. Thirdly, patterns of shale gas migration play an important role in describing shale gas percolation accurately. It is found that the classic Darcy percolation law cannot accurately describe shale gas behavior especially in nanopores and under different gas states so that desorption, diffusion and slippage, viscous flow of shale gas in pores, Knudsen diffusion and surface diffusion of adsorbed gas, adsorption/desorption, matrix-fracture transfer, and non-Darcy effects [15-20] are introduced to describe the percolation law of shale gas; then, the percolation effect between matrix and fractures through percolation velocity, reservoir pressure, and production was analyzed. Fourthly, the improvement of the mathematical calculation method is another key point, such as the lattice Boltzmann method (LBM), finite element method, and finite volume method $[14,21,22]$ which are introduced to improve the accuracy of gas percolation representation considering adsorptive/cohesive forces between the molecules and fracture network distribution. Finally, the boundary layer, stress sensitivity, and many other properties of a reservoir and fluid can affect the micropercolation of shale gas $[23,24]$.

In summary, previous studies have mainly focused on the occurrence state of shale gas, multiscale pore structure, percolation patterns and percolation mathematical model, analytical methods, and other properties to study shale gas percolation mainly from two aspects in productivity and reservoir pressure. But few people have interpreted the shale gas percolation law from the perspective of gas molecules, and gas viscosity properties from the molecular level to analyze shale gas percolation were ignored. And most importantly, under high reservoir pressure, the pressure is the main factor affecting the viscosity of the gas. Therefore, a mathematical model of gas percolation, considering the effect of reservoir pressure on gas viscosity in matrix-fracture system-dual media after fracturing, was established based on previous research results. The effect of gas viscosity on shale gas percolation was studied by comparing and analyzing the reservoir pressure, gas percolation velocity, and bottom hole flowing pressure in the near well section in this work.

\section{Modeling}

2.1. Establishment of Physical Model. Hydraulic fracturing technology was adopted in a horizontal well of a shale gas reservoir. According to its symmetry, the reservoir area above the horizontal wellbore was selected for research. Three-stage staged fracturing technology is adopted, and the single-well simulation domain in the reservoir is $400 \mathrm{~m}$ $(L) \times 240 \mathrm{~m}(W / 2) \times 38 \mathrm{~m}\left(T_{h}\right)$. The interaction criterion between artificial fracture and natural fracture under high stress and high approximation angle is that artificial fractures go directly through natural fractures and keep going in the same direction $[25,26]$. The artificial fracture and natural fracture are perpendicular to each other in this paper, and a typical matrix-fracture system is formed in the fracture network region. After hydraulic fracturing, the physical model is shown in Figure 1.

\subsection{Mathematical Model of Percolation}

2.2.1. Basic Assumptions. The following assumptions are put forward based on the double-porosity physical model. Firstly, gas percolation channels in shale reservoirs are mainly matrix and fractures, and fractures are discontinuous and discrete. Secondly, the shale gas reservoir is isotropic, and its fluid only contains compressible single-phase gas. However, the reservoir is slightly compressible, and the compressibility does not change with time. Thirdly, it is isothermal during gas flow. Fourthly, the influence of capillary pressure and gravity is not considered during gas flow. Fifthly, the gas flow in the matrix and fracture systems is in accordance with the Darcy flow. Sixthly, both the free gas and dissolved gas in the initial shale gas reservoir are ignored, and the free gas in the matrix and fractures is from the desorption of adsorbed gas in the matrix system. Seventhly, gas migrated from the distal end reservoir to the fracture zone and finally flows into the wellbore through artificial fractures, ignoring the gas directly flowing into the wellbore from the matrix and natural fractures. Eighthly, the gas well's imperfection is ignored.

2.2.2. Percolation Mathematical Model of Matrix System. The continuity equation of the matrix system considering adsorbed gas is established as follows:

$$
\frac{\partial}{\partial t}\left(\phi_{m} \rho_{\mathrm{gm}}+\rho_{s} V_{E}\right)=-\operatorname{div}\left(\rho_{\mathrm{gm}} v_{m}\right)-q
$$

where $\phi_{m}$ is the porosity of the matrix system, $\rho_{\mathrm{gm}}$ is the gas density in the matrix system, $V_{E}$ is the adsorption quantity of shale gas, $\rho_{s}$ is the gas density at standard condition, $v_{m}$ is gas percolation velocity in the matrix system, and $q$ is the flow of interfacial flow between matrix and fractures.

Substitute the equation of motion $v_{m}=-\left(K_{m} / \mu\right) \operatorname{grad} P_{m}$ , the equation of state $\phi_{m}=\phi_{\text {mo }}+C_{m}\left(P_{m}-P_{i}\right)$, the equation of real gas $\rho_{\mathrm{gm}}=P M / Z R T$, the equation of state for desorption gas $V_{E}=V_{m}\left(P / P_{\mathrm{L}}+P\right)$, the equation of gas compressibility $C_{\mathrm{gm}}=1 / P_{m}-(1 / Z)\left(\partial Z / \partial P_{m}\right)$, and the equation of interfacial flow $q=\left(a K_{m} / \mu\right) \rho_{o}\left(P_{m}-P_{f}\right)$ into Equation (1), then it can be got as follows:

$$
\begin{gathered}
\frac{M P_{m}}{Z R T}\left[C_{m}+C_{\mathrm{gm}} \phi_{\mathrm{mo}}+C_{m} C_{\mathrm{gm}}\left(P_{m}-P_{i}\right)+\frac{\rho_{s} V_{m} P_{\mathrm{L}}}{\left(P_{\mathrm{L}}+P_{m}\right)^{2}}\right] \frac{\partial P_{m}}{\partial t} \\
=-\frac{M K_{m}}{R T} \operatorname{div}\left(\frac{P_{m}}{Z \mu} \nabla P_{m}\right)-\frac{a K_{m}}{\mu} \rho_{o}\left(P_{m}-P_{f}\right),
\end{gathered}
$$

where $M$ is the molecular weight of shale gas, $Z$ is the compressibility factor, $R$ is the universal gas constant, $T$ is the 


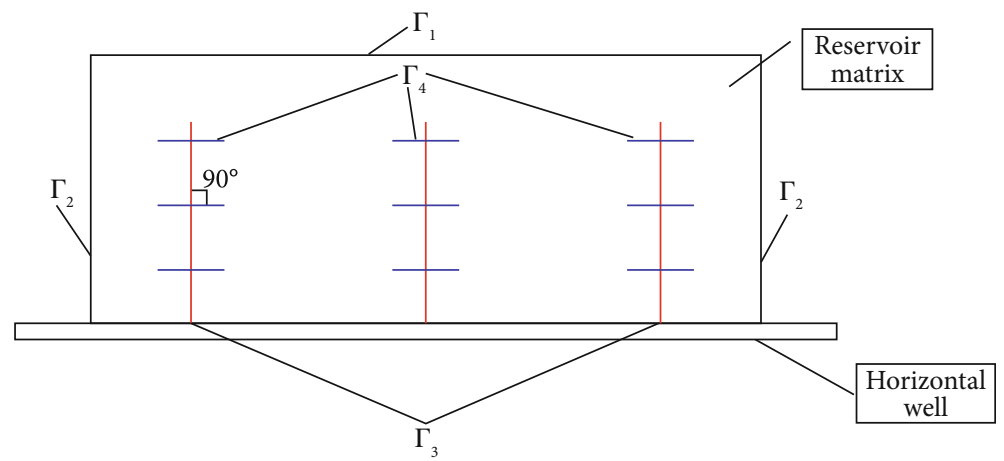

Figure 1: Double-porosity physical model. $\Gamma_{1}$ and $\Gamma_{2}$ : reservoir boundary; $\Gamma_{3}$ : artificial fracture; $\Gamma_{4}$ : natural fracture.

reservoir temperature, $P_{m}$ is the pressure of the matrix system, $P_{\mathrm{L}}$ is the Langmuir pressure constant, $V_{m}$ is the Langmuir volume, $C_{m}$ is the pore compressibility of the matrix system, $C_{\mathrm{gm}}$ is the gas compressibility of the matrix system, $K_{m}$ is the permeability of the matrix system, $\Phi_{\text {mo }}$ is the initial porosity of the matrix system, $\mu$ is the gas viscosity in the reservoir, $a$ is the shape factor, and $\rho_{o}$ is the density of shale gas at the pressure of $P_{i}$.

Because both $C_{m}$ and $C_{\mathrm{gm}}$ are of smaller order of magnitude, $C_{m} C_{\mathrm{gm}}$ can be ignored; Equation (2) can be simplified as follows:

$$
\begin{aligned}
\frac{P_{m} \phi_{\mathrm{mo}}}{Z}\left[C_{\mathrm{gm}}+\frac{C_{m}}{\phi_{\mathrm{mo}}}+\frac{\rho_{s} V_{m} P_{L}}{\rho_{\mathrm{gm}} \phi_{\mathrm{mo}}\left(P_{L}+P_{m}\right)^{2}}\right] \frac{\partial P_{m}}{\partial t} \\
=-K_{m} \operatorname{div}\left(\frac{P_{m}}{Z \mu} \nabla P_{m}\right)-\frac{R T}{M} \frac{a K_{m}}{\mu} \rho_{o}\left(P_{m}-P_{f}\right) .
\end{aligned}
$$

Let the total compressibility of the matrix system be $C_{\mathrm{tm}}=C_{\mathrm{gm}}+C_{m} / \phi_{\mathrm{mo}}+\rho_{s} V_{m} P_{L} / \rho_{\mathrm{gm}} \phi_{\mathrm{mo}}\left(P_{L}+P_{m}\right)^{2}$, then Equation (3) can be simplified as follows:

$\frac{P_{m} C_{\mathrm{tm}} \phi_{\mathrm{mo}}}{Z} \frac{\partial P_{m}}{\partial t}=-K_{m} \operatorname{div}\left(\frac{P_{m}}{Z \mu} \nabla P_{m}\right)-\frac{R T}{M} \frac{a K_{m}}{\mu} \rho_{o}\left(P_{m}-P_{f}\right)$

The known condition is $\psi_{m}=\int_{0}^{P_{m}}(2 P / \mu Z) d p$; then, substitute the known condition into Equation (4); then, it can be got as follows:

$$
\frac{\partial \psi_{m}}{\partial t}=-\frac{K_{m}}{\phi_{\mathrm{mo}} \mu C_{\mathrm{tm}}} \operatorname{div}\left(\nabla \psi_{m}\right)-2 a \frac{K_{m}}{\phi_{\mathrm{mo}} \mu C_{\mathrm{tm}}}\left(\psi_{m}-\psi_{f}\right) .
$$

When the gas viscosity is related to pressure, $\mu C_{\text {tm }}$ cannot be treated as a constant, and the pseudotime $t_{a}=\int_{0}^{t}\left(1 / \mu C_{\mathrm{tm}}\right.$ )$d t$ is introduced to linearize the equation. So, Equation (5) can be simplified as follows:

$$
\frac{\partial \psi_{m}}{\partial t_{a}}=-\frac{K_{m}}{\phi_{\mathrm{mo}}} \operatorname{div}\left(\nabla \psi_{m}\right)-2 a \frac{K_{m}}{\phi_{\mathrm{mo}}}\left(\psi_{m}-\psi_{f}\right) .
$$

Since the coefficient $K_{m} / \phi_{\text {mo }}$ is a constant, $2 a\left(K_{m} / \phi_{\text {mo }}\right)$ is also a constant. Let the coefficients $K_{m} / \phi_{\text {mo }}$ and $2 a\left(K_{m} / \phi_{\text {mo }}\right)$ be $a_{1}$ and $a_{2}$, substitute them into Equation (6), and the percolation mathematical model of the matrix system can be got as follows:

$$
\frac{\partial \psi_{m}}{\partial t_{a}}=-a_{1} \operatorname{div}\left(\nabla \psi_{m}\right)-a_{2}\left(\psi_{m}-\psi_{f}\right)
$$

2.2.3. Percolation Mathematical Model of Fracture System. Continuity equation of fracture system:

$$
\frac{\partial}{\partial t}\left(\phi_{f} \rho_{g f}\right)=-\operatorname{div}\left(\rho_{\mathrm{gf}} v_{f}\right)+q
$$

where $\phi_{f}$ is the porosity of the fracture system, $\rho_{\text {gf }}$ is the gas density of the fracture system, and $v_{f}$ is gas percolation velocity in the fracture system.

Substitute equation of motion $v_{f}=-\left(K_{f} / \mu\right) \operatorname{grad} P_{f}$, equation of state $\phi_{f}=\phi_{\mathrm{fo}}+C_{f}\left(P_{f}-P_{i}\right)$, compressibility coefficient $C_{\mathrm{gf}}=1 / P_{f}-(1 / Z)\left(\partial Z / \partial P_{f}\right)$ of the fracture system, and the equation of interfacial flow $q=\left(a K_{m} / \mu\right) \rho_{o}\left(P_{m}-P_{f}\right)$ into Equation (8), then it can be simplified as follows:

$$
\begin{aligned}
\frac{\phi_{\mathrm{fo}} P_{f}}{Z}\left[\frac{C_{f}}{\phi_{\mathrm{fo}}}+C_{\mathrm{gf}}+\frac{C_{f} C_{\mathrm{gf}}\left(P_{f}-P_{i}\right)}{\phi_{\mathrm{fo}}}\right] \frac{\partial P_{f}}{\partial t} \\
=-K_{f} \operatorname{div}\left(\frac{P_{f}}{Z \mu} \operatorname{grad} P_{f}\right)+\frac{R T}{M} \frac{a K_{m}}{\mu} \rho_{o}\left(P_{m}-P_{f}\right),
\end{aligned}
$$

where $P_{f}$ is the pressure of the fracture system, $C_{f}$ is the pore compressibility of the fracture system, $C_{\mathrm{gf}}$ is the gas compressibility of the fracture system, $K_{f}$ is the permeability of the fracture system, and $\Phi_{\text {fo }}$ is the initial porosity of the fracture system. 
Because the order of magnitude of $C_{f}$ and $C_{\mathrm{gf}}$ is small, $C_{f} C_{\mathrm{gf}}$ can be ignored, and let equation $C_{\mathrm{tf}}=C_{\mathrm{gf}}+C_{f} / \phi_{\mathrm{fo}}$ be the total compressibility of the fracture system, then Equation (9) continues to be simplified as follows:

$\frac{\phi_{\mathrm{fo}} P_{f} C_{\mathrm{tf}}}{Z} \frac{\partial P_{f}}{\partial t}=-K_{f} \operatorname{div}\left(\frac{P_{f}}{Z \mu} \operatorname{grad} P_{f}\right)+\frac{R T}{M} \frac{a K_{m}}{\mu} \rho_{o}\left(P_{m}-P_{f}\right)$.

The known condition is $\psi_{f}=\int_{0}^{P_{f}}(2 P / \mu Z) d p$; then, substitute it into Equation (10); then, the equation can be simplified as follows:

$$
\frac{\partial \psi_{f}}{\partial t}=-\frac{K_{f}}{\phi_{\mathrm{fo}} \mu C_{\mathrm{tf}}} \operatorname{div}\left(\nabla \psi_{f}\right)+2 a \frac{K_{f}}{\phi_{\mathrm{fo}} \mu C_{\mathrm{tf}}}\left(\psi_{m}-\psi_{f}\right) .
$$

When considering that the gas viscosity is related to pressure, $\mu C_{\mathrm{tf}}$ cannot be treated as a constant; the pseudotime $t_{b}=\int_{0}^{t}\left(1 / \mu C_{\mathrm{tf}}\right) d t$ is introduced to linearize the equation. So, Equation (11) can be simplified as follows:

$$
\frac{\partial \psi_{f}}{\partial t_{b}}=-\frac{K_{f}}{\phi_{\mathrm{fo}}} \operatorname{div}\left(\nabla \psi_{f}\right)+2 a \frac{K_{f}}{\phi_{\mathrm{fo}}}\left(\psi_{m}-\psi_{f}\right) .
$$

Since the coefficient $K_{f} / \phi_{\text {fo }}$ is a constant, $2 a\left(K_{f} / \phi_{\text {fo }}\right)$ is also a constant. Let the coefficients $K_{f} / \phi_{\text {fo }}$ and $2 a\left(K_{f} / \phi_{\text {fo }}\right)$ be $b_{1}$ and $b_{2}$, substitute them into Equation (12), and the percolation mathematical model of the fracture system can be got as follows:

$$
\frac{\partial \psi_{f}}{\partial t_{b}}=-b_{1} \operatorname{div}\left(\nabla \psi_{f}\right)+b_{2}\left(\psi_{m}-\psi_{f}\right) .
$$

2.2.4. Boundary and Original Conditions. Both the boundary conditions and the initial conditions were constructed to satisfy the discrete fractured reservoir according to the actual production of the gas field and physical model.

(1) Outer Boundary Condition: Constant Pressure.

$$
\psi_{m}(x, y, t) \mid \Gamma_{1}=\psi_{o}(x, y, t),(x, y) \in \Gamma_{1} .
$$

(2) Inner Boundary Condition: Constant Production.

$$
\begin{gathered}
\psi_{m}(x, y, t) \mid \Gamma_{1}=\psi_{o}(x, y, t),(x, y) \in \Gamma_{1}, \\
\frac{\partial \psi(x, y, t)}{\partial n} \mid \Gamma_{3}=\frac{2 Q P}{K A Z},(x, y) \in \Gamma_{3} .
\end{gathered}
$$

(3) Initial Condition. When the shale gas reservoir is not opened, reservoir pressure is the original reservoir pressure as follows:

$$
\psi_{m}(x, y, t=0)=\psi_{f}(x, y, t=0)=\psi_{o}(x, y, t=0) .
$$

(4) Inner Boundary Conditions at the Fracture.

$$
\begin{gathered}
\psi_{m}(x, y, t)=\psi_{f}(x, y, t) \mid \Gamma_{4},(x, y) \in \Gamma_{4}, \\
K_{m} \frac{\partial \psi_{m}}{\partial n}\left|\Gamma_{4}=K_{f} \frac{\partial \psi_{f}}{\partial l}\right| \Gamma_{4},(x, y) \in \Gamma_{4} .
\end{gathered}
$$

\section{Model Solution}

\subsection{Basic Parameters}

3.1.1. Shale Gas Reservoir and Gas Basic Parameters. The well depth is $2000 \mathrm{~m}$, and the borehole radius is $0.1 \mathrm{~m}$. There are 3 artificial fractures and 9 natural fractures. The reservoir and gas parameters involved in the solution process of the model are partly from the experimental and production data of the shale gas reservoir of the Longmaxi formation in southern Sichuan as shown in Table 1.

\subsubsection{Viscosity Parameter Processing}

(1)When Viscosity Is Not a Constant. The main component of shale gas is methane [29-31]. Therefore, the viscosity of methane can be used to replace the shale gas viscosity. The known condition is that the methane viscosity is $0.0135 \mathrm{mPa} \cdot \mathrm{s}$ under $0.1 \mathrm{MPa}$ and $333.15 \mathrm{~K}$. And the critical pressure and critical temperature of methane are $4.59 \mathrm{MPa}$ and $190.55 \mathrm{~K}$, respectively [32]:

$$
\begin{gathered}
T_{\mathrm{pr}}=\frac{T}{T_{\mathrm{pc}}}=\frac{333.15}{190.55} \approx 1.75, \\
P_{\mathrm{pr}}=\frac{P}{P_{\mathrm{pc}}}=\frac{P}{4.59}(4.59 \mathrm{MPa} \leq P \leq 30 \mathrm{MPa}),
\end{gathered}
$$

where $T$ is the absolute temperature of gas, $T_{\mathrm{pc}}$ is critical temperature of gas, $T_{\mathrm{pr}}$ is corresponding temperature, $P$ is gas pressure, $P_{\mathrm{pc}}$ is critical pressure of gas, and $P_{\mathrm{pr}}$ is the corresponding pressure, $P_{\mathrm{pr}} \in[1,6.5]$.

Refer to the dimensionless viscosity, corresponding pressure, and temperature chart published by Herning and L. Zipperer [33], then the dimensionless viscosity $\mu_{g} / \mu_{g 1}$ was obtained according to the corresponding temperature $T_{\mathrm{pr}}($ 1.75) and corresponding pressure $P_{\text {pr }}$ calculated by Equation (18). Meanwhile, $\mu_{\mathrm{g} 1}$ is the viscosity of methane, and its viscosity is known to be $0.0135 \mathrm{mPa} \cdot \mathrm{s}$ under $0.1 \mathrm{MPa}$ and $333.15 \mathrm{~K}$. So, a large number of viscosity points and reservoir pressure points were obtained; then, the viscosity points and reservoir pressure points were fitted under the condition of greater than critical pressure of methane and $333.15 \mathrm{~K}$; the relationship between viscosity and reservoir pressure can be got as Figure 2.

According to the fitting curve shown in Figure 2, viscosity and pressure of methane satisfy the linear relationship as follows:

$$
\mu=0.0004 P+0.0119
$$


TABLE 1: Basic parameters of shale reservoir and shale gas $[27,28]$.

\begin{tabular}{|c|c|c|c|}
\hline Symbol & Value & Unit & Description \\
\hline$T$ & 333.15 & $\mathrm{~K}$ & Reservoir temperature \\
\hline$L$ & 400 & $\mathrm{~m}$ & Length of reservoir \\
\hline$W$ & 480 & $\mathrm{~m}$ & Width of reservoir \\
\hline$T_{h}$ & 38 & $\mathrm{~m}$ & Thickness of reservoir \\
\hline$W_{\mathrm{NF}}$ & 0.002 & $\mathrm{~m}$ & Natural fracture width \\
\hline$W_{\mathrm{AF}}$ & 0.005 & $\mathrm{~m}$ & Artificial fracture width \\
\hline$K_{m}$ & $5.622 \times 10^{-6}$ & $\mu \mathrm{m}^{2}$ & Matrix permeability \\
\hline$K_{\mathrm{nf}}$ & $1.5 \times 10^{-5}$ & $\mu \mathrm{m}^{2}$ & Natural fracture permeability \\
\hline$K_{\mathrm{af}}$ & $2.5 \times 10^{-2}$ & $\mu \mathrm{m}^{2}$ & Artificial fracture permeability \\
\hline$C_{m}$ & $3 \times 10^{-4}$ & $\mathrm{MPa}^{-1}$ & Matrix compressibility \\
\hline$C_{\mathrm{nf}}$ & $2.5 \times 10^{-4}$ & $\mathrm{MPa}^{-1}$ & Natural fracture compressibility \\
\hline$C_{\mathrm{af}}$ & $4 \times 10^{-4}$ & $\mathrm{MPa}^{-1}$ & Artificial fracture compressibility \\
\hline$\Phi_{\mathrm{mo}}$ & 2 & $\%$ & Matrix porosity \\
\hline$\Phi_{\text {nfo }}$ & 0.4 & $\%$ & Natural fracture porosity \\
\hline$\Phi_{\mathrm{afo}}$ & 0.4 & $\%$ & Artificial fracture porosity \\
\hline$P_{o}$ & 30 & $\mathrm{MPa}$ & Initial reservoir pressure \\
\hline AS & 3 & - & Artificial fracture number \\
\hline$L_{\mathrm{AF}}$ & 200 & $\mathrm{~m}$ & Half length of artificial fracture \\
\hline$Q$ & 3000 & $\mathrm{~m}^{3} / \mathrm{d}$ & Half gas flow rate \\
\hline$\rho_{\mathrm{GD}}$ & 0.717 & $\mathrm{~kg} / \mathrm{m}^{3}$ & Gas density at standard conditions \\
\hline$\rho_{\mathrm{UD}}$ & 176.794 & $\mathrm{~kg} / \mathrm{m}^{3}$ & Gas density at reservoir conditions \\
\hline$V_{m}$ & 2 & $\mathrm{~m}^{3} / \mathrm{t}$ & Langmuir volume \\
\hline$P_{\mathrm{L}}$ & 10 & $\mathrm{MPa}$ & Langmuir pressure \\
\hline$R$ & 8.34 & $\mathrm{~J} /(\mathrm{K} \cdot \mathrm{mol})$ & Universal gas constant \\
\hline$M$ & 16 & $\mathrm{~g} / \mathrm{mol}$ & Molecular weight of methane \\
\hline$a$ & 0.76 & $\mathrm{~m}^{-2}$ & Shape factor \\
\hline
\end{tabular}

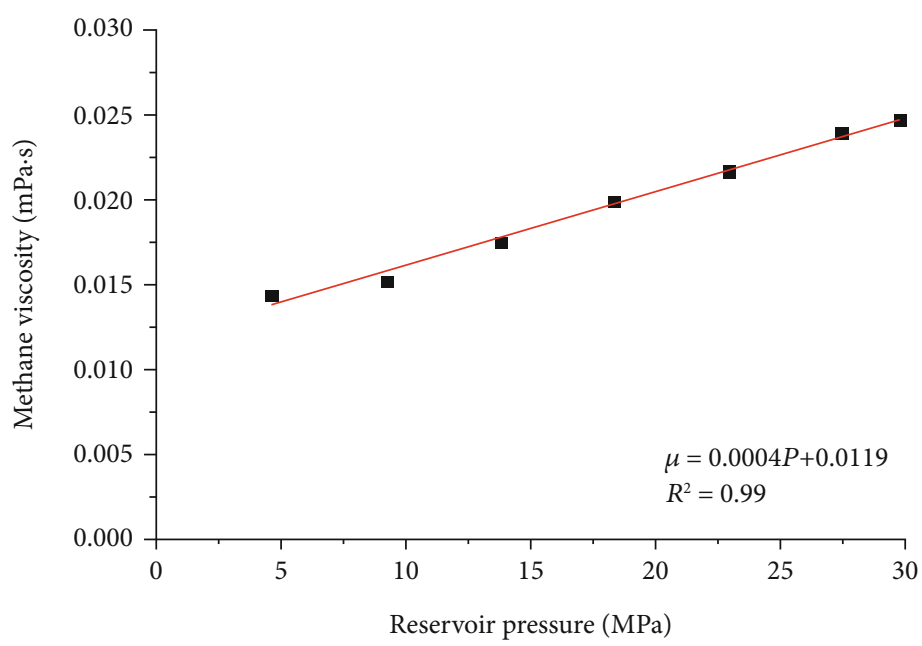

FIGURE 2: Viscosity of methane changes over pressure. 


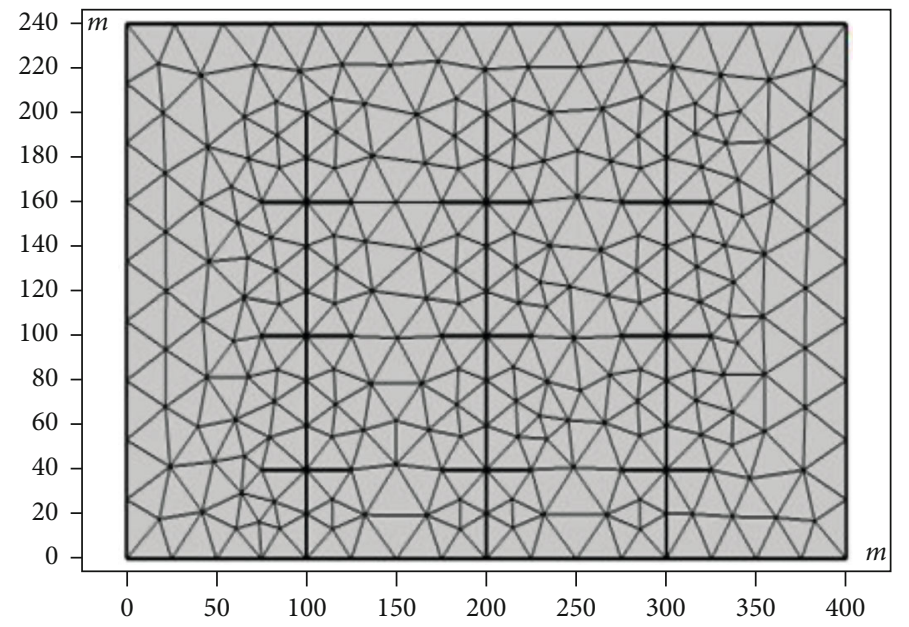

FiguRE 3: Free triangular mesh result.

where $P$ is reservoir pressure and $\mu$ is the viscosity of methane.

(2) When Viscosity Is a Constant. Substitute reservoir temperature $333.15 \mathrm{~K}$ and pressure $30 \mathrm{MPa}$ into the fitting equation and the approximate value of methane viscosity is got as follows:

$$
\mu=0.0239 \mathrm{mPa} \cdot \mathrm{s} \text {. }
$$

3.2. Model Solution. Based on the mathematical model of shale gas percolation in dual media and combining with basic reservoir and gas parameters, the reservoir pressure, percolation velocity, and bottom hole flowing pressure under different shale gas viscosities were, respectively, solved by the finite element method. Free triangular mesh (as shown in Figure 3 ) is used as the computing grid to solve the solution region. The distribution nephograms of reservoir pressure, velocity field, and the curve of bottom hole flowing pressure changing with time from $0 \mathrm{~d}$ to $1000 \mathrm{~d}$ were drawn and compared separately.

3.2.1. Distribution Nephograms of Reservoir Pressure and Velocity Field. For shale gas with low viscosity, the velocity of gas percolation and reservoir pressure are greatly affected by viscosity under high reservoir pressure. As shown in Figures 4 and 5, the reservoir pressure and the shale gas velocity change over time under the effect of gas viscosity. By comparing Figures 4(a), 4(b), 5(a), and 5(b), it shows that firstly, the pressure drops more rapidly and obviously in the fracture system, while the pressure in the matrix system hardly changes, and there is no big difference in pressure change under different gas viscosities, so the gas viscosity change has little effect on reservoir pressure distribution. Secondly, shale gas velocity in the fracture system is obviously faster than that in the matrix system, and gas percolation velocity is much faster in fracture systems when pressure has an effect on gas viscosity than that when pressure has no effect on gas viscosity. Thirdly, the minimum isobaric value is $1.6 \mathrm{MPa}$ when pressure affects shale gas viscosity.
However, the minimum isobaric value is $1.4 \mathrm{MPa}$ when pressure does not affect shale gas viscosity. The phenomena above can be explained as follows: due to the high conductivity of the fracture system and extremely low permeability of the matrix system, a large number of free shale gas migrated from fracture system to horizontal wellbore rapidly at first and pressure drops more rapidly and obviously in the fracture system than that in the matrix system when shale gas is extracted at a fixed production capacity. Meantime, when pressure has an effect on gas viscosity, the gas viscosity decreases with reservoir pressure decreasing; gas percolation velocity is much faster than that when pressure has no effect on gas viscosity that remains a high constant as the reservoir opens [34].

By comparing Figures 4(c) and 4(d) and Figures 5(c) and 5(d), it shows that the isobaric lines were gradually densified and much denser, the pressure spreads to a wider area, and pressure drop is much noticeable in the matrix system than those when pressure has no effect on gas viscosity at the middle stage. These phenomena can be explained as follows: due to the production of free shale gas in the fracture system, the desorption of adsorbed shale gas in the matrix system occurred at the far end of the well and begins to supplement the fracture system. When pressure has an effect on gas viscosity, the gas migration velocity is increased rapidly so that the pressure drop rate, pressure wave propagation velocity, and range are increased, which is more conducive to the desorption of adsorbed shale gas in the matrix system, and the desorption amount is larger than those when pressure has no effect on gas viscosity [35].

By comparing Figures 4(e) and 4(f)and Figures 5(e) and $5(\mathrm{f})$, it shows that the pressure and velocity distributions in the fracture and matrix systems almost remain a stable state at the late stage. These phenomena can be explained as follows: gas viscosity keeps constant due to the reservoir pressure so that gas migration velocity remains unchanged, so the amount of shale gas adsorption and desorption reached a balance in the matrix system basically.

3.2.2. Reservoir Pressure Near Wellbore. Each curve in Figure 6 represents the bottom hole flowing pressure with 


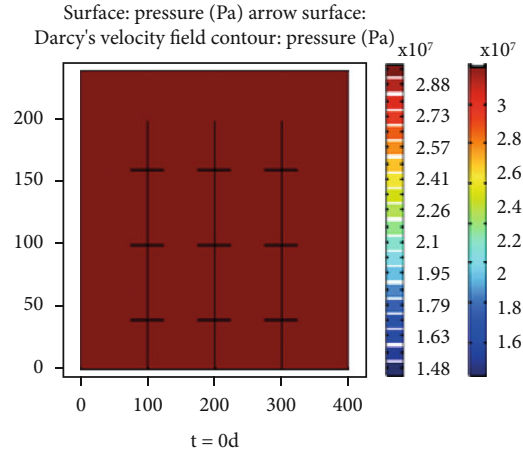

(a) $t=0 \mathrm{~d}$

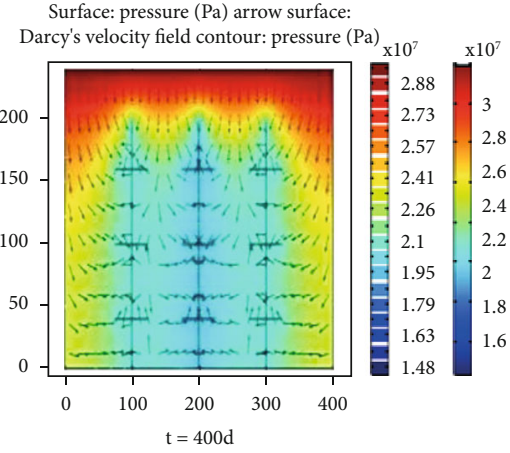

(c) $t=400 \mathrm{~d}$

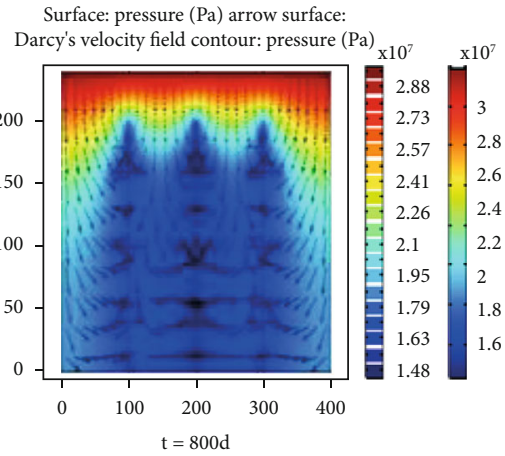

(e) $t=800 \mathrm{~d}$

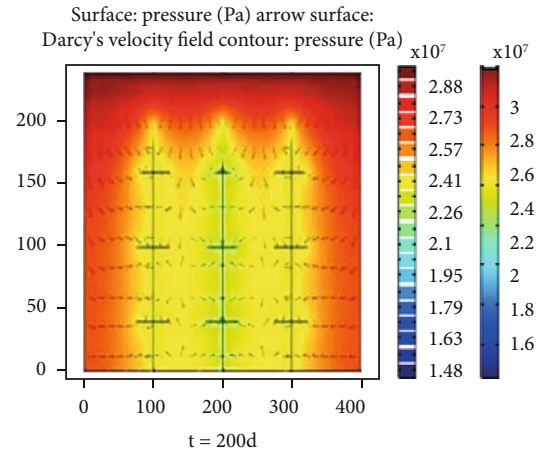

(b) $t=200 \mathrm{~d}$

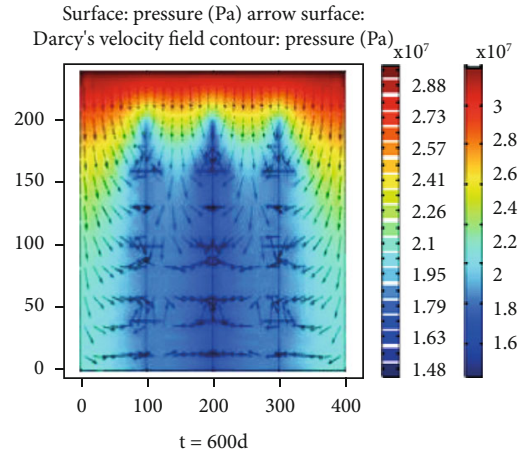

(d) $t=600 \mathrm{~d}$

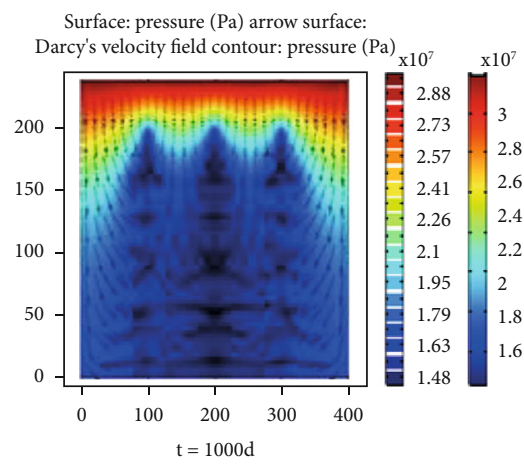

(f) $t=1000 \mathrm{~d}$

FIGURE 4: Distribution nephograms of reservoir pressure and velocity field over time under nonconstant gas viscosity.

different gas viscosities under different exploitation stages. It shows that gas viscosity change hardly has an effect on bottom hole flowing pressure in the early stage (Stage 1) of production. When the reservoir opens, a large amount of free shale gas was mainly produced from the fracture system, the desorption amount of the adsorbed gas in the matrix system is relatively less, and its contribution to bottom hole flowing pressure is much smaller, so the bottom hole flowing pressure decreases quickly and there was no significant difference in pressure between different gas viscosities.

As the production enters the middle stage (Stage 2), the free shale gas in the fracture system gradually decreases, which makes the adsorbed shale gas in the matrix system desorb to supplement the formation pressure gradually and slow down the bottom hole flowing pressure drop rate, but the viscosity differences of shale gas make a significant difference in bottom hole flowing pressure. When pressure affects shale gas viscosity, gas viscosity decreases with reservoir pressure reducing; both the desorption amount of shale gas in the matrix system and the gas percolation velocity are increased to supplement the bottom hole flowing pressure quickly, which slows down the bottom hole flowing pressure drop rate gradually and shortens the gas migration time to the horizontal wellbore. However, when pressure has no effect on shale gas viscosity, gas viscosity remains unchanged with reservoir pressure decreasing; shale gas still flows at a high viscosity, which causes that percolation velocity is relatively slow. But most importantly, it not only is conducive to the desorption of adsorbed gas in the matrix system but also prolongs the gas migration time to the horizontal wellbore. 


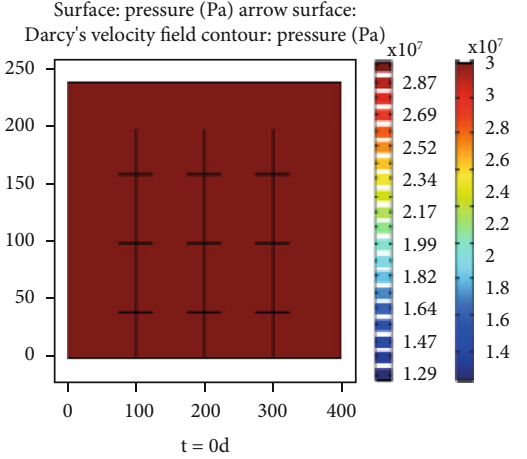

(a) $t=0 \mathrm{~d}$

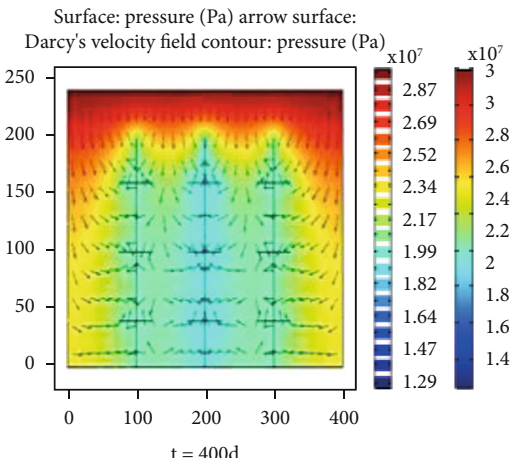

(c) $t=400 \mathrm{~d}$

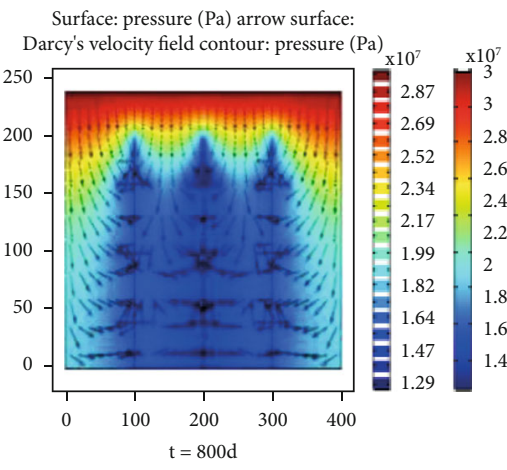

(e) $t=800 \mathrm{~d}$

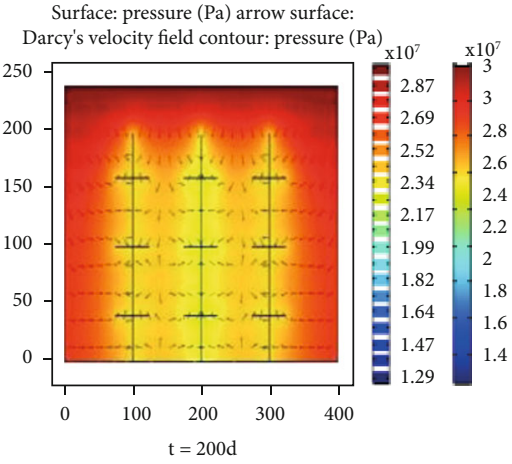

(b) $t=200 \mathrm{~d}$

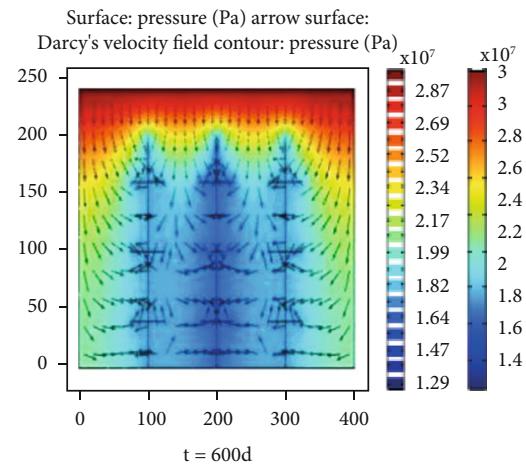

(d) $t=600 \mathrm{~d}$

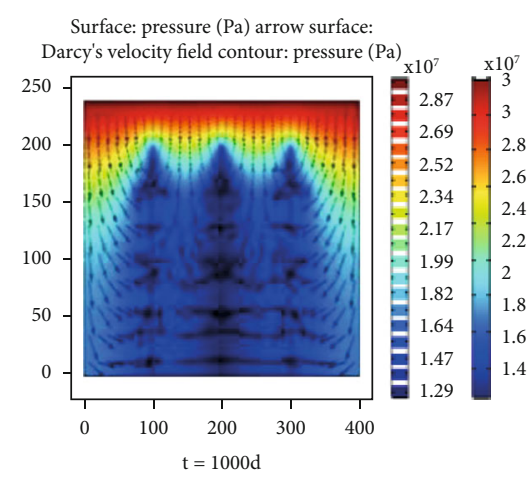

(f) $t=1000 \mathrm{~d}$

FIGURE 5: Distribution nephograms of reservoir pressure and velocity field over time under constant gas viscosity.

Therefore, the bottom hole flowing pressure is much higher, and the pressure drop range is much larger than that when reservoir pressure has no effect on gas viscosity.

As the production enters the late stage (Stage 3), the adsorption and desorption capacity of shale gas in the matrix system reach a balance. So, when shale gas is extracted at a fixed production capacity, the reservoir pressure remains unchanged, the gas viscosity basically does not change, and the percolation velocity remains unchanged, which makes the bottom hole flowing pressure under different shale gas viscosities tend to be stable.

\section{Conclusions}

In conclusion, a double-porosity mathematical model, which is based on unsteady percolation theory of single-phase slightly compressible fluid, was established considering the gas occurrence state and the effect of pressure on gas viscosity, and the role of gas viscosity for shale gas percolation was studied by comparing reservoir pressure, percolation velocity, and bottom hole flowing pressure. The result shows that gas viscosity has significant effect on gas percolation and gas desorption. The following details were drawn:

(1) When pressure affects the viscosity of shale gas, the viscosity of shale gas decreased with reservoir pressure decreasing, and it was much smaller than that when pressure did not affect the gas viscosity. Both percolation velocity of free shale gas and the reservoir velocity of pressure drop in the matrix and fracture systems are increased, and the two are the fastest in the fracture system. Meanwhile, the increasing of 


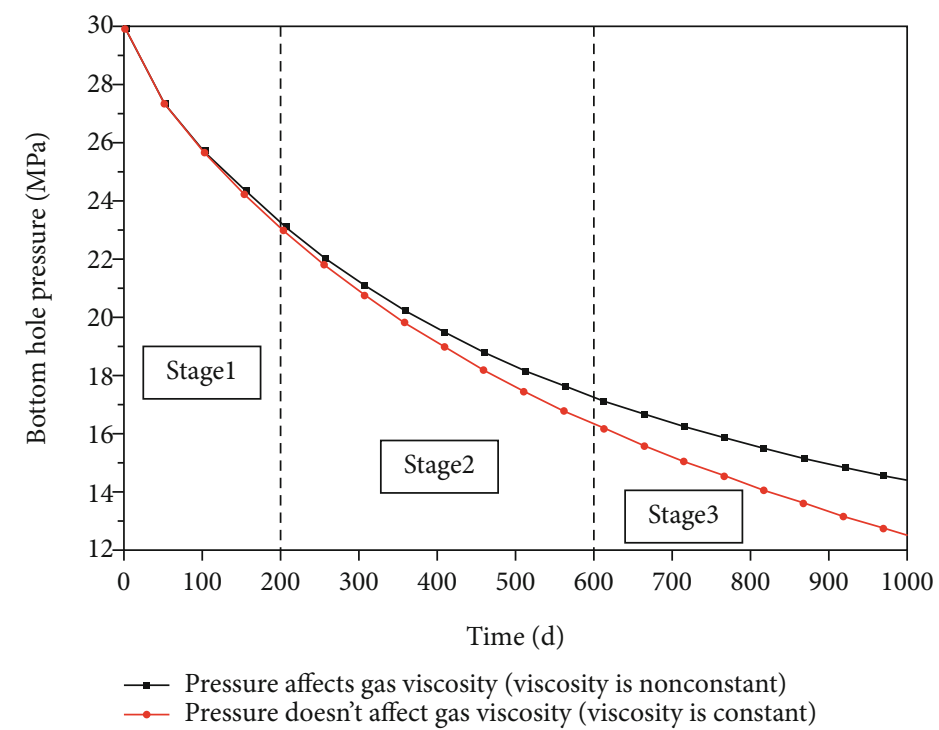

FIGURE 6: Bottom hole flowing pressure changes with time under different gas viscosities. Stage 1: early stage; Stage 2: middle stage; Stage 3: late stage.

pressure wave propagation speed and range is conducive to the desorption of adsorbed shale gas at the far end of the horizontal well, and the desorption amount is relatively large

(2) When pressure affects the viscosity of shale gas, the viscosity of shale gas decreased, which increased the percolation velocity of shale gas, so that the matrix system pressure decreased relatively fast. This shortens the time for gas migration to the horizontal wellbore and for wellbore pressure to reach stability. With free shale gas in the fracture system being extracted, the free shale gas in the fracture decreased, shale gas in the matrix system comes into play, and the desorption of adsorbed shale gas in the matrix system replenishes formation pressure with the exploitation of free shale gas in the fracture system. And also, the desorption amount of shale gas increased with the increase of pressure difference, which makes the bottom hole flowing pressure decrease slowly, and higher than that pressure has no effect on gas viscosity

This work helps in understanding the mechanism of shale gas percolation with viscosity changing, which will benefit the production of shale gas in unconventional tight shale reservoirs.

\section{Data Availability}

The data used to support the findings of this study are available from the corresponding author upon request.

\section{Conflicts of Interest}

The authors declare that they have no conflicts of interest.

\section{Acknowledgments}

The project was supported by the National Natural Science Foundation of China (Project Nos. 51874239 and 51804254).

\section{References}

[1] A. M. Jaffe, "Shale Gas Will Rock the World," in Alternative Energy and Shale Gas Encyclopedia, J. H. Lehr, J. Keeley, and T. B. Kingery, Eds., Wiley, 2016.

[2] U S Energy Information Administration (EIA), “Technically Recoverable Shale Oil," in Shale Gas Resources: An Assessment of 137 Shale Formations in 41 Countries Outside the United States, EIA report, 2013.

[3] J. D. Arthur, B. Langhus, and D. Alleman, "An overview of modern shale gas development in the United States," All Consulting, vol. 3, pp. 14-17, 2018.

[4] Y. Tang, X. Tang, G. Y. Wang, and Q. Zhang, "Summary of hydraulic fracturing technology in shale gas development," Geological Bulletin of China, vol. 30, no. z1, pp. 393-399, 2011.

[5] D. X. Zhang and T. Y. Yang, "An overview of shale-gas production," Acta Petrolei Sinica, vol. 34, no. 4, pp. 792-801, 2013.

[6] J. Yao, H. Sun, and A. F. Li, "Modern system of multiphase flow in porous media and its development trend," Chinese Science Bulletin, vol. 63, no. 4, pp. 425-451, 2017.

[7] H. Sun, A. Chawathe, H. Hoteit, X. Shi, and L. Li, "Understanding shale gas flow behavior using numerical simulation," SPE Journal, vol. 20, no. 1, pp. 142-154, 2015.

[8] A. F. Li, Petrophysics, China University of Petroleum Press, Dongying, 3rd edition, 2011.

[9] F. Javadpour, "Nanopores and apparent permeability of gas flow in mudrocks (shales and siltstone)," Journal of Canadian Petroleum Technology, vol. 48, no. 8, pp. 16-21, 2013.

[10] W. Y. Zhu, Q. Qi, M. A. Qian, M. Yue, and Y. Z. Liu, "Unstable seepage modeling and pressure propagation of shale gas reservoirs," Petroleum Exploration and Development, vol. 43, no. 2, pp. 261-267, 2016. 
[11] W. Q. Liu, D. N. Wang, and Q. Su, "Dual media model of shale layer with anisotropy involved and its simulation on gas migration," Natural Gas Geoscience, vol. 27, no. 8, pp. 13741379, 2016.

[12] L. D. Mi, H. Q. Jiang, T. Li, and J. J. Li, "Characterization and dynamic analysis of shale gas production based on discrete fracture model," Journal of China University of Petroleum (Edition of Natural Science), vol. 39, no. 3, pp. 126-131, 2015.

[13] D. Fan and A. Ettehadtavakkol, "Semi-analytical modeling of shale gas flow through fractal induced fracture networks with microseismic data," Fuel, vol. 193, pp. 444-459, 2017.

[14] C. Tang, X. F. Chen, Z. du, P. Yue, and J. B. Wei, "Numerical simulation study on seepage theory of a multi-section fractured horizontal well in shale gas reservoirs based on multiscale flow mechanisms," Energies, vol. 11, no. 9, article 2329, 2018.

[15] X. Guo and W. F. Wang, "Seepage mechanism and transient pressure analysis of shale gas," Applied Mathematics, vol. 4, no. 1, pp. 197-203, 2013.

[16] J. Deng, W. Y. Zhu, and Q. Ma, "A new seepage model for shale gas reservoir and productivity analysis of fractured well," Fuel, vol. 124, pp. 232-240, 2014.

[17] J. Deng, W. Y. Zhu, Q. Qi, W. Tian, and M. Yue, "Study on the steady and transient pressure characteristics of shale gas reservoirs," Journal of Natural Gas Science and Engineering, vol. 24, pp. 210-216, 2015.

[18] W. Y. Zhu and Q. Qi, "Study on the multi-scale nonlinear flow mechanism and model of shale gas," Scientia Sinica (Technologica), vol. 46, no. 2, pp. 111-119, 2016.

[19] Y. Xia, Y. Jin, M. Chen, and K. P. Chen, "Gas flow in shale reservoirs," Chinese Science Bulletin, vol. 60, no. 24, pp. 22592271, 2015.

[20] B. Gong, G. Qin, B. F. Towler, and H. Y. Wang, "Discrete modeling of natural and hydraulic fractures in shale-gas reservoirs," in SPE Annual Technical Conference and Exhibition, pp. 1-9, Denver, Colorado, USA, 2011.

[21] E. Fathi and I. Y. Akkutlu, "Lattice Boltzmann method for simulation of shale gas transport in kerogen," SPE Journal, vol. 18, no. 1, pp. 27-37, 2012.

[22] I. Y. Akkutlu, Y. Efendiev, M. Vasilyeva, and Y. H. Wang, "Multiscale model reduction for shale gas transport in a coupled discrete fracture and dual-continuum porous media," Journal of Natural Gas Science and Engineering, vol. 48, pp. 65-76, 2017.

[23] G. I. Barenblatt, P. J. Monteiro, and C. H. Rycroft, "On a boundary layer problem related to the gas flow in shales," Journal of Engineering Mathematics, vol. 84, no. 1, pp. 11-18, 2014.

[24] Y. Li, P. C. Dong, and W. R. Li, "The gas flow in micro fractures of shale gas reservoirs," in SPE Middle East Oil \& Gas Show and Conference, pp. 1-15, Manama, Kingdom of Bahrain, 2017.

[25] T. L. Blanton, "Propagation of hydraulically and dynamically induced fractures in naturally fractured reservoirs," in SPE Unconventional Gas Technology Symposium, pp. 1-15, Louisville, Kentucky, 1986.

[26] S. Y. Chen, W. H. Li, L. L. Du, and B. Song, "Research on the acting criteria when hydraulic fracture intersects with natural fracture," Sino-Global Energy, vol. 19, no. 10, pp. 37-43, 2014.

[27] M. Gao, X. F. Xian, Y. G. Du, and Y. Y. Lu, "The inorganic composition, structure and adsorption properties of the shale cores from the Weiyuan gas reservoirs, Sichuan Basin," Natural Gas Industry, vol. 32, no. 6, pp. 106-109, 2012.

[28] X. Z. Guo and C. S. Zhou, "Seepage numerical model for fractured horizontal well in shale gas reservoir," Journal of Southwest Petroleum University (Science \& Technology Edition), vol. 36, no. 5, pp. 90-96, 2014.

[29] Z. Chen, L. Chen, G. C. Wang et al., "Applying isotopic geochemical proxy for gas content prediction of Longmaxi shale in the Sichuan Basin, China," Marine and Petroleum Geology, vol. 116, p. 104329, 2020.

[30] J. X. Dai, C. N. Zou, S. M. Liao et al., "Geochemistry of the extremely high thermal maturity Longmaxi shale gas, southern Sichuan Basin," Organic Geochemistry, vol. 74, pp. 3-12, 2014.

[31] Z. Q. Feng, S. P. Huang, W. Wu, C. Xie, W. Peng, and Y. Cai, "Longmaxi shale gas geochemistry in Changning and Fuling gas fields, the Sichuan Basin," Energy Exploration \& Exploitation, vol. 35, no. 2, pp. 259-278, 2017.

[32] Sinopec Shanghai Engineering Co Ltd, Hua Gong Gong Yi She Ji Shou Ce (in Chinese), Chemical Industry Press, 3rd edition, 2009.

[33] A. F. Li, You Ceng Wu Li Xue (in Chinese), China University of Petroleum Press, Dong ying, 3rd edition, 2011.

[34] H. Kazemi, "Pressure transient analysis of naturally fractured reservoirs with uniform fracture distribution," Society of Petroleum Engineers Journal, vol. 9, no. 4, pp. 451-462, 2013.

[35] F. J. Kuchuk, "Radius of investigation for reserve estimation from pressure transient well tests," in SPE Middle East Oil and Gas Show and Conference, pp. 1-21, Manama, Bahrain, 2009. 